Military Technical College Kobry El-Kobbah, Cairo, Egypt

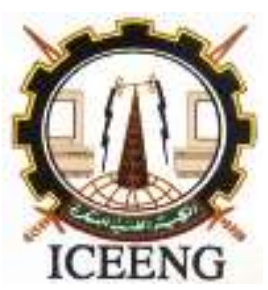

\author{
$1^{\text {th }}$ International Conference \\ on Electrical Engineering \\ ICEENG 2018
}

\title{
SIMO-OFDM Cellular Network: Multiuser Interference Mitigation and Desired User Identification
}

\author{
Ehab M. Shaheen*, M.Samir *, and Ahmed A. Amin *
}

\section{Abstract :}

The rapidly increasing demand for mobile broadband traffic drives the need for even more capacity from cellular networks. Multiple Input Multiple Output (MIMO) technology is a key element of modern wireless communication standard including Long Term Evolution (LTE) and WiMAX. MIMO systems are used to improve the robustness of data transmission, to increase data rate, to improve capacity and other aspects of network performance. In the absence of line of sight (LOS) between the mobile receiver station (MS) and the base station (BS), the localization of the BS is very important. The Direction of Arrival (DOA) algorithms can estimate the incident angles of all the received signals impinging on the array. These algorithms give the DOAs of all the relevant signals of the user sources and interference sources. Yet, they are not capable of distinguish and identify which one is the direction of the desired user. In this paper, a reference signal is known by the transmitter and the receiver is proposed to identify which one of the estimated DOAs is the direction of the desired user in the cell. With the aid of such reference signal and applying the correlation concept, the DOA algorithms can distinguish the direction of the desired signal from the others. This concept was applied to the MUltiple SIgnal Classification (MUSIC), ROOT MUSIC, and Minimum Variance Distortion less Response (MVDR) algorithms. Moreover, the Affine Projection Algorithm (APA) is classified as a fast adaptive filter was implemented in this paper to enhance the accuracy of the estimated direction and to form a beam towards the desired user and nulls towards interferes. Extensive simulation results assure that in the presence of the proposed reference signal, the DOAs algorithms can identify the direction of the desired user with high accuracy and resolution.

* $\quad$ Egyptian Armed Forces 


\section{Keywords:}

Affine Projection Algorithm; Direction of Arrival; Minimum Variance Distortion less Response Algorithm; MUSIC; Root MUSIC; and Adaptive Beam-forming.

\section{Introduction:}

The challenging requirements of future wireless broadband communications are to achieve high spectral efficiency along with high transmission rate [1]. Multiple Input Multiple Output (MIMO) systems lead to the achievement of high data rate without increasing the total transmission power or bandwidth [2]. In practice, wireless communication channels may encounter frequency selectivity and time variation especially for broadband and mobile applications. MIMO can be used along with orthogonal frequency division Multiplexing (OFDM) to improve the performance of broadband communication standard systems such as LTE and WiMAX over which the impact of frequency selective fading channel can be reduced [3].

The rapid growth in mobile communication users necessitates the development of reliable communication systems that provide higher data rates. The space division multiple Access (SDMA) is the key element that can enable reusing of the same channels among different users in the call. Initially, it is required to identify the user's position in the cell and secondly, to cancel the interference signals from the adjacent cellular network. The existing DOA algorithms have the ability to estimate the direction of the incoming signals impinging on the antenna array elements [4]. Yet, unfortunately they cannot distinguish among which signal belongs to which users and consequently, the direction of the desired user cannot be accurately estimated.

The transmission of the reference signals between the base station and the user is very vital in mobile communication to identify the users in the cellular network. It can be defined as a sequence of bits which are known by both the transmitter and the receiver. There are two main types of reference signal in modern communication systems such as LTE [5]. The first type is the downlink reference signal which provides the mobile with the amplitude and phase reference signal in order to estimate the channel status indicator (CSI) and calculate the channel quality indicator (CQI) consequently. The second type is the uplink reference signal which is used in channel estimation procedure done by base stations.

DOA techniques have been used in a wide range of applications such as navigation, radar, and tracking objects. They can be classified into four categories [6]. The first one is the conventional DOA techniques where beam-forming and null steering is used to estimate the DOAs. Capon's minimum variance is an example of these conventional 
techniques [7]. The second category is the subspace based techniques. These techniques build the DOA estimation based on the received data. There are two main algorithms of this category [8]. The first algorithm is the MUSIC algorithm which is based on the decomposition of the eigenvalues of the estimated correlation matrix of the received signal. The second algorithm is the Estimation of Signal Parameters via Rotational Invariance Technique (ESPRIT) which uses the signal subspace to estimate the DOAs [9]. The third category is the Maximum Likelihood (ML) techniques. These techniques have high computational complexities which make them less popular.

Noted that ML techniques have advantages over other techniques in the cases of low Signal to Noise Ratio (SNR) levels and highly correlated signals. The last category is the integrated techniques which mix the subspace-based techniques with the property of restoral.

Adaptive beam forming techniques are used for enhancing the desired users signal while suppressing the noise and the interference at the array output by using adaptive smart antenna systems. The radiation pattern is dynamically controlled to perform a beam steering to the desired user direction and generating a null steering toward the interfering signal [10].

In [11] the Affine Projection Algorithm (APA) was implemented to enhance the accuracy of the estimated direction due to its fast convergence rate, fast tracking and low steady state error and a continuation to this work the APA algorithm is used in this paper as an adaptive beam forming algorithm to generate a beam towards the desired user in the cellular network and nulls towards the others.

The main contributions of this paper can be summarized as follows:

- The cross correlation concept is applied to the single input multiple output (SIMO) OFDM system with the aid of a reference signal to identify the DOA of the desired user in the cell.

- An antenna's beam-forming algorithm was implemented in order to suppress the interfering signals from the desired user signal. This is achieved by generating a main beam towards the desired user direction and nulls towards the other interfering signals.

- The impact of low SNR on the accuracy of the MVDR, MUSIC, and the Root MUSIC algorithms at different SNR below $0 \mathrm{~dB}$ is investigated.

The rest of this paper is organized as following. Section II presents the SIMO-OFDM system model and introduces the DOA Algorithms. In section III the cross correlation concept is analytically derived. Section IV introduces the accuracy performance enhancement. Simulation results are presented and verified in section V before drawing the conclusions in section VI.

\section{System Model:}


The received signal impinging on a uniform linear array (ULA) consisting of $M$ identical and omnidirectional antenna elements with an incident angle $\theta$ is shown in Fig. 1. Block 1 represents the DOA estimation Algorithm and block 2 represents the adaptive beam forming algorithm.

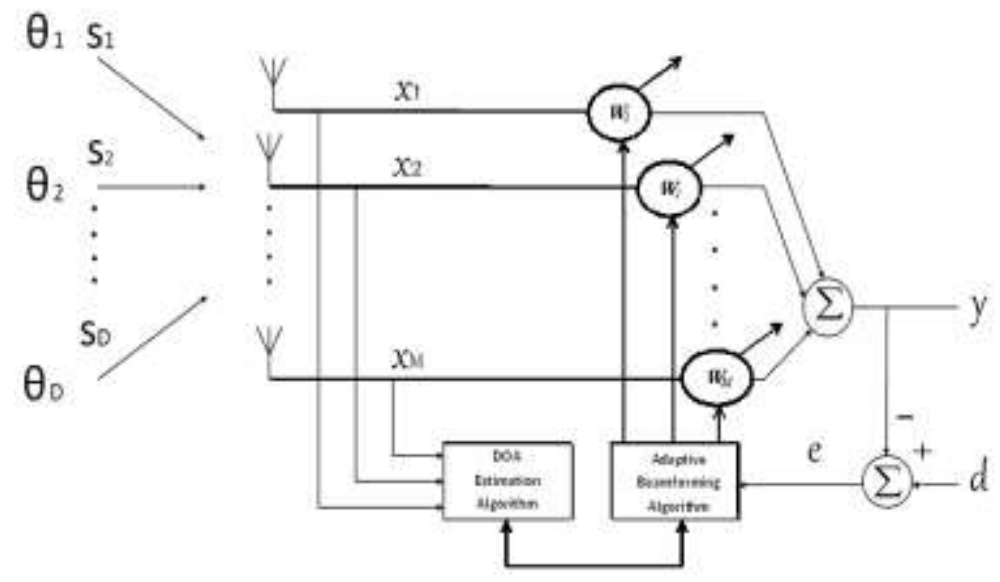

Figure (1): OFDM antenna receiver model for D users.

This signal will arrive at the $M^{\text {th }}$ elements with delay equals to

$\Delta_{m}=[(m-1) \cdot d \sin (\theta)] / C$

Where $C$ the speed of light and $\mathrm{d}$ is the distance between two adjacent antenna array elements.

Consider $D$ numbers of signals are arriving to the antenna. The steering vector of the antenna can be expressed as [12].

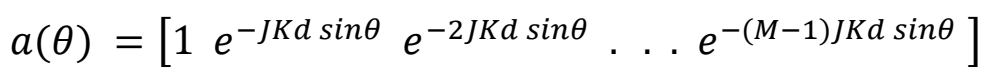

Where $K$ is the wave length number.

The received signal by the antenna array elements can be expressed as

$x(t)=\left[\begin{array}{c}x_{1}(t) \\ x_{2}(t) \\ \cdot \\ \cdot \\ \cdot \\ x_{D}(t)\end{array}\right]=\left[\begin{array}{llll}a\left(\theta_{1}\right) & a\left(\theta_{2}\right) & \ldots & a\left(\theta_{D}\right)\end{array}\right]\left[\begin{array}{c}S_{1}(t) \\ S_{2}(t) \\ \cdot \\ \cdot \\ \cdot \\ S_{M}(t)\end{array}\right]+n(t)$

Which can be simplified into

$x(t)=A \cdot S(t)+n(t)$

Where $\boldsymbol{x}(t)$ is the data column vector received by the array, $\boldsymbol{S}(t)$ is the signal column vector generator by the sources and $\boldsymbol{n}(t)$ is a zero mean additive white noise vector. The 
development of the DOA estimation schemes is essentially can be achieved when forming and steering the array in one direction at a time and measure the output power. When the steered direction coincides with the DOA of a signal then the maximum output power will be observed [13].

A weight vector $\boldsymbol{W}$ can be designed and then used to combine the data received by the array elements to form a single output signal $\boldsymbol{y}(t)$, which can be written as

$y(t)=W^{H} x(t)$

Where $H$ is the Hermitian operator.

The total averaged output power out of an array over $N$ snapshots can be expressed as

$$
\begin{aligned}
P(W) & =\frac{1}{N} \sum_{n=1}^{N}\left|y\left(t_{n}\right)\right|^{2} \\
& =\frac{1}{N} \sum_{n=1}^{N} W^{H} x\left(t_{n}\right) x\left(t_{n}\right)^{H} W \\
& =W^{H} \hat{R}_{x x} W
\end{aligned}
$$

Where $\widehat{\boldsymbol{R}}_{x x}$ is the estimate of the data covariance matrix $\mathbf{R}_{x x}$.

The array's response to the incoming signals can be controlled by adjusting the elements of weight vector $\boldsymbol{W}$. This process is referred to as spatial filtering or beamforming. This fundamental data matrix $\widehat{\boldsymbol{R}}_{x x}$ is used in all our description of DOA estimation algorithms. Many DOA estimation algorithms basically try to extract the information from this array data covariance matrix such as Minimum Variance Distortion less Response (MVDR), multiple signal classification (MUSIC) and Root MUSIC algorithms.

\section{Mathematical Correlation Model:}

One way to estimate the DOAs is to scan through the set of all possible steering vectors. Once the signals are separated with respect to their distinct DOAs, the desired user corresponding to these DOAs needs to be identified. For D signals, $S_{i}$ signals transmitted at angles $\theta_{i}$, where $i=1,2, \ldots, \mathrm{D}$.

The steering vector of the $S_{i}$ signals received by the elements of the antenna array can be expressed as

$$
a\left(\theta_{i}\right)=\left[\begin{array}{lllll}
1 & e^{-J K d \sin \theta_{i}} & e^{-2 J K d \sin \theta_{i}} & \cdots & e^{-(M-1) J K d \sin \theta_{i}}
\end{array}\right]
$$

The received signals by the antenna elements will be corrupted due to the presence of noise and the effect of the Rayleigh fading channels. Thus, the $i^{\text {th }}$ received signals can be written as

$x_{i}=a^{H}\left(\theta_{i}\right) S_{i}\left(\theta_{i}\right)+n$ 
Furthermore, the true value of received angle will be deviated from the actual transmitted ones. Therefore these signals will be received at angles $\hat{\theta}_{\text {est }_{i}}$, and the steering vectors according to these estimated angles can be written as

$a\left(\hat{\theta}_{e s t_{i}}\right)=\left[\begin{array}{lll}1 & e^{-J K d \sin \widehat{\theta}_{e s t_{1}}} e^{-2 J K d \sin \widehat{\theta}_{\text {est }}} \cdots e^{-(M-1) J K d \sin \widehat{\theta}_{e s t_{i}}}\end{array}\right]$

Where, $\boldsymbol{a}\left(\hat{\theta}_{\text {est }_{i}}\right)$ is the new steering vector according to the estimated DOAs algorithm.

Noted that, a threshold level is considered to limit the number of the resultant peaks in the MUSIC and MVDR algorithms. Yet, there is no need for it in the case of Root MUSIC.

The received data by the $i^{\text {th }}$ array antenna element from a single output can be written as follows

$y_{i}(t)=W^{H} x_{i}(t)$

Where $\boldsymbol{W}$ represents the $M \times 1$ complex weighting vector and can be used to steer the array response to the direction of the incoming signals and can be written as

$W=a\left(\hat{\theta}_{e s t_{i}}\right)=\left[\begin{array}{lllll}1 & e^{-J K d \sin \hat{\theta}_{e s t_{1}}} & e^{-2 J K d \sin \hat{\theta}_{e s t_{2}}} \cdots e^{-(M-1) J K d \sin \hat{\theta}_{e s t_{i}}}\end{array}\right]$

Then from equations (9) and (10), the output of the antenna array elements can be expressed as

$y_{i}(t)=a^{H}\left(\hat{\theta}_{e s t_{i}}\right) x_{i}(t)$

Then, the decision statistics for the transmitted signals can be constructed as

$\hat{S}_{i}(t)=\sum_{i=1}^{D} a^{-1}\left(\hat{\theta}_{\text {est }}\right) * y_{i}(t)$

Where $\widehat{\boldsymbol{S}}_{i}$ represents the transmitted signals at the estimated directions obtained from the peaks resultant from the DOA algorithms.

The desired user signal needs to be distinguished from the other received signals. Thus, one can use the cross correlation concept among these estimated signals received by the antenna array elements and the reference signal which is known to the transmitter and the receiver. This cross correlation concept is used to identify which one from the resultant peaks obtained by the DOA algorithms is the desired user. To this end, this cross correlation can be written as

$$
Z_{i}(t)=\int_{-\infty}^{\infty} f^{*}(t) . \hat{S}_{i}(t+\tau) d t
$$

where, $f^{*}(t)$ denotes the complex conjugate of the reference signal $f(t)$ and $\tau$ is the lag. This cross correlation concept can be used to estimate how much the estimated 
transmitted signals $\widehat{\boldsymbol{S}}_{i}$ and the reference signal are correlated and finally from that one can get

$\max (\operatorname{corr}(i))=\max \left(Z_{i}(\tau)\right)$

From equation (14) as a result, the signal which has the largest correlation with the reference signal can be considered as the desired user signal and its steering vector can be used to represent the estimated desired user direction.

\section{Accuracy Enhancement Performances:}

The Affine Projection Algorithm (APA) is an adaptive filter algorithm that utilizes the gradient based method. This method employs the error vector instead of a scalar error which is used in Least Mean Square algorithm to adjust the variable step size [14]. The Wiener filter coefficient can be written as [15]

$\widehat{d}(n)=\sum_{k=0}^{p} h(k) x(n-k)$

Where $\boldsymbol{h}(k)$ is the unit sample response of the finite impulse response (FIR) Wiener filter that process $\boldsymbol{d}(n), \boldsymbol{x}(n)$.

The error signal can be estimated as

$e(n)=d(n)-\widehat{d}(n)=d(n)-\sum_{k=0}^{p} h(k) x(n-k)$

Where $e(n)$ is the error between the desired user signal and the actual output from the Wiener filter.

The coefficients of the Wiener filter are obtained by minimizing the MSE which is considered as the optimum criteria for beam-forming and it can be written as

$E\left\{|e(n)|^{2}\right\}=E\left\{\left|\widehat{d}(n)-\sum_{k=0}^{p} W(k) x(n-k)\right|^{2}\right\}$

Then by solving the Wiener-Hopf, one can get

$R_{x x} W=r_{x d}$

where $\boldsymbol{R}_{x x}$ is the covariance matrix of the input data matrix to the filter and $\boldsymbol{r}_{x d}$ is the cross-correlation vector between the input data vector $\boldsymbol{x}(k)$ and the training sequence $d(n)$ result after using the filter coefficients to converge the data to their describe values. The coefficient vector recursion can be written as

$\boldsymbol{h}(n+1)=\boldsymbol{h}(n)+\mu(n) \boldsymbol{x}(n)\left(\boldsymbol{x}^{T}(n) \boldsymbol{x}(n)+\delta_{1} I\right)^{-1}$ 
Where $\delta_{1}$ is a small positive number, I is a unit matrix of size $M \times M$ and $\boldsymbol{x}(n)$ is the received signal.

Then the variable step size $\mu(n)$ can be obtained as

$\mu(n)=\mu_{\max } \frac{\|P(n)\|^{2}}{\|P(n)\|^{2}+\delta_{2}}$

where $\delta_{2}$ is a positive number proportional to $M$ and inversely proportional to SNR, $\mu_{\text {max }} \leq 2$ and $P(n)$ is an $M \times 1$ vector recursively. $P(n)$ can be estimated as

$P(n)=\beta P(n+1)+(1-\beta) \boldsymbol{x}(n)\left(\boldsymbol{x}^{T}(n) \boldsymbol{x}(n)+\delta_{1} I\right)^{-1} e(n)$

where $\beta$ is a smoothing factor, $0 \leq \beta \leq 1$.

\section{Simulation Results:}

In this section the performance of the DOA algorithms (MUSIC, Root MUSIC and MVDR) under the effect of frequency selective fading channels is investigated. The considered uniform linear array (ULA) is composed of ten elements. The enter spacing between the antenna elements is $d=0.5 \lambda$. A Quadrature Phase Shift Keying is considered to modulate snapshots composed of one sample each. Four OFDM signals (with parameter according to the IEEE802.11a standard) impinging on the antenna array have been considered arriving at angles $\left[\begin{array}{llll}-60^{\circ} & -30^{\circ} & 30^{\circ} & 60^{\circ}\end{array}\right]$ respectively. Initially the ability of the MUSIC and the MVDR algorithms to estimate the direction of the desired user in terms of the reference signal at different SNR values is investigated. Figure (2) depicts how the MUSIC and the MVDR algorithms scan the angle $\theta$ over the angular region of the interest for the ULA from $-90^{\circ}$ to $+90^{\circ}$ degrees and measure the output power of the steered array to find out the peaks of the incoming signal at different SNR $(0 \mathrm{~dB}$ and $-10 \mathrm{~dB})$ values.

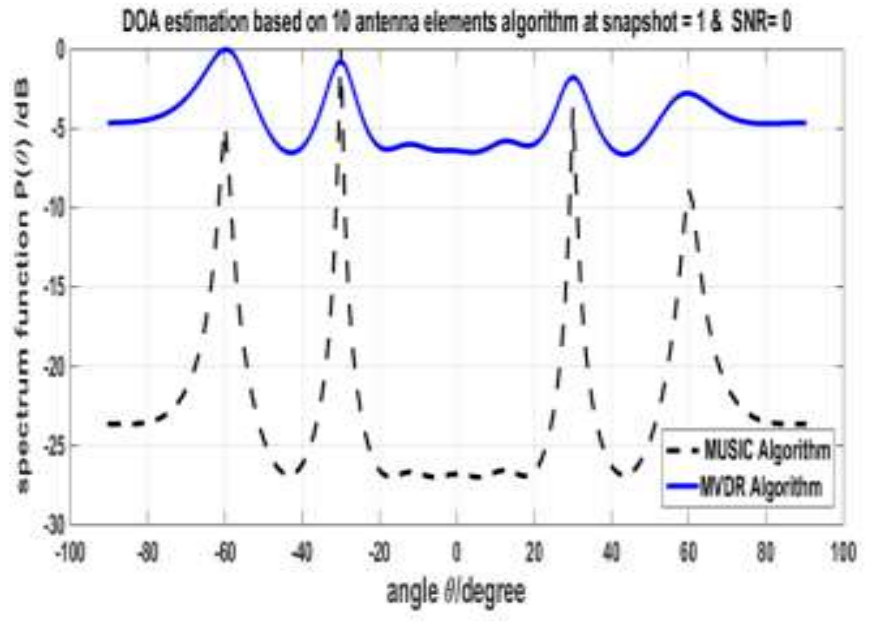

a. $S N R=O d B$

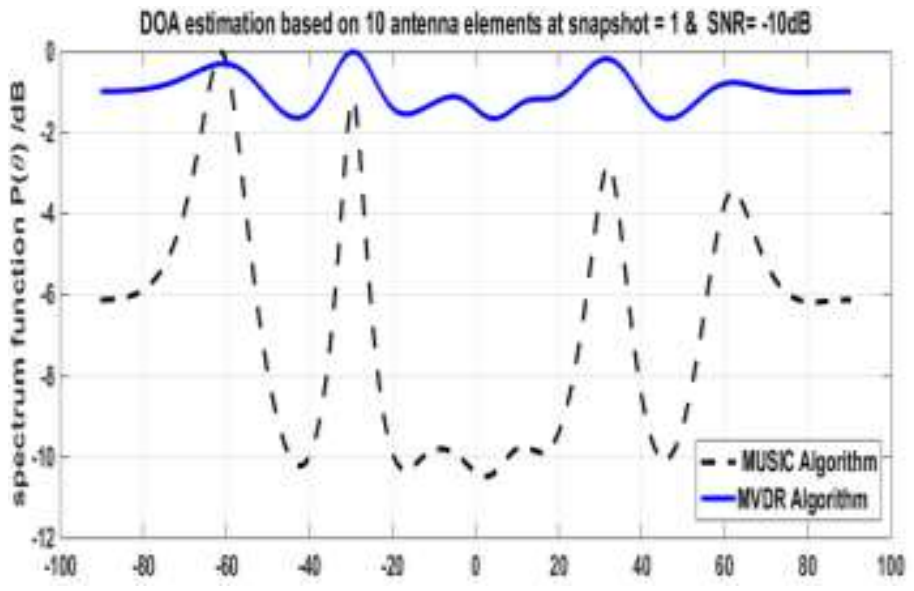

b. $S N R=-10 d B$ 
Figure (2): DOA estimation comparison of MUSIC and MVDR algorithms for 10

elements ULA at one snapshot.

It can be seen that at values less than $0 \mathrm{~dB}$ as shown in figure (2b) the performance of the MUSIC and the MVDR algorithms are degraded causing an accuracy error of the measured angles of the incoming signals. Also, it was observed that the MUSIC algorithm outperforms the MVDR for SIMO-OFDM signals using four transmitted antennas from four users and 10 elements receiving antenna array.

Table 1 summarizes the obtained estimated angles for the four mentioned OFDM signals by the MUSIC and MVDR algorithms at $\mathrm{SNR}=0 \mathrm{~dB}$ and $\mathrm{SNR}=-10 \mathrm{~dB}$. It was observed that after a threshold level is considered to limit the number of the resultant peaks, the MVDR algorithm estimates more interference signal than the exact transmitted signals (four OFDM transmitted signals) at both the SNR values. Yet, the MUSIC algorithm estimates more interference signals at $\mathrm{SNR}=-10 \mathrm{~dB}$. The performance of both the MUSIC and the MVDR algorithm is degrades at SNR below $0 \mathrm{~dB}$.

Table (1): MUSIC and MVDR algorithms Results

\begin{tabular}{|c|c|c|c|c|c|c|c|}
\hline DOA Algorithm & SNR & \multicolumn{6}{|c|}{ Estimated Angles ( $\widehat{\boldsymbol{\theta}}_{\text {est }}$ ) } \\
\hline \multirow{2}{*}{ MVDR } & $0 \mathrm{~dB}$ & $-60^{\circ}$ & $-30^{\circ}$ & $-10^{\circ}$ & $-4^{\circ}$ & $30^{\circ}$ & $60^{\circ}$ \\
\hline & $-10 \mathrm{~dB}$ & $-56^{\circ}$ & $-35^{\circ}$ & $-22^{\circ}$ & $-3^{\circ}$ & $25^{\circ}$ & $66^{\circ}$ \\
\hline \multirow{2}{*}{ MUSIC } & $0 \mathrm{~dB}$ & $-60^{\circ}$ & $-30^{\circ}$ & - & - & $30^{\circ}$ & $60^{\circ}$ \\
\hline & $-10 \mathrm{~dB}$ & $-59^{\circ}$ & $-28^{\circ}$ & $-14^{\circ}$ & $1^{\circ}$ & $33^{\circ}$ & $55^{\circ}$ \\
\hline
\end{tabular}

The second step is to generate a new steering vector according to the estimated DOAs. Then, by applying cross correlation between the detected signals received by the antenna array elements and the reference signal, it was found that the desired user is located at the direction of $+33^{\circ}$. Now, this information of the new steering vector at the obtained direction of the desired user was fed as initial weights to the APA algorithm. This is done firstly to enhance the accuracy of the DOA of the desired user direction measured by the DOA algorithm. Secondly, after that enhancing the DOA result by adjusting the adaptive weights of the adaptive APA beam forming filter to generate a beam in the direction of the desired user and nulls towards the interferes.

Figure (3) depicts how the APA algorithm gets the optimum weights after less than 100 iterations where the error is reduced to the minimum. 


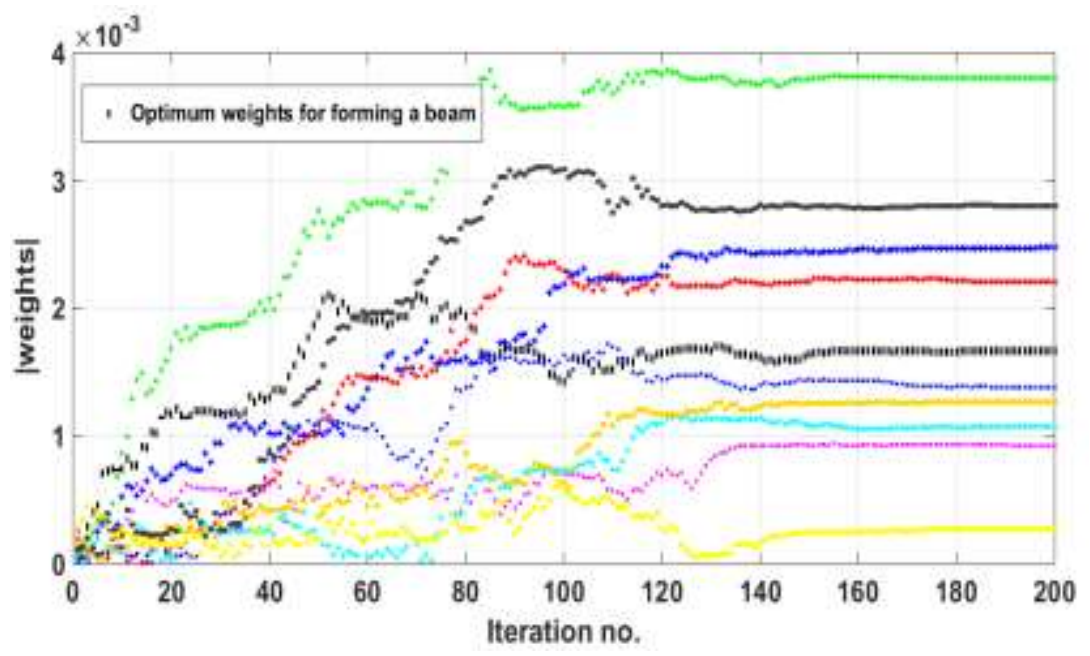

Figure (3): Optimum weights after applying the fast adaptive APA algorithm.

Figures (4a) and (4b) depict how the APA algorithm forms the main beam towards the desired user and nulls towards the interfere signals in Cartesian and Polar coordinates respectively where, the APA improves the DOA accuracy by adjusting the weights of the antenna elements with fast convergence rate, fast tracking and low steady state error. Figures (5a) and (5b) depict the behavior of the Root MUSIC algorithm at SNR $=0 \mathrm{~dB}$ and $-10 \mathrm{~dB}$ respectively. The Root MUSIC algorithm figures out the estimated signals by examining the roots of the spectrum polynomial whose coefficients are elements of a vector in the noise subspace. Eight signals are present corresponding to the eight roots that lie on the unit circle and ten noise component at $\mathrm{SNR}=0 \mathrm{~dB}$ as shown in figure (5a) but at $\mathrm{SNR}=-10 \mathrm{~dB}$, the roots lie away from the unit circle as shown in figure $(5 b)$.

Table (2): Root MUSIC algorithm Result

\begin{tabular}{|c|c|c|c|c|c|c|}
\hline Algorithm & SNR & \multicolumn{5}{|c|}{ Estimated Angles ( $\left.\widehat{\boldsymbol{\theta}}_{\text {est }}\right)$} \\
\hline \multirow{4}{*}{ Root MUSIC } & \multirow{2}{*}{$0 \mathrm{~dB}$} & $\pm 37.93^{\circ}$ & $\pm 40.08^{\circ}$ & $\pm 12.71^{\circ}$ & $\pm 0.49^{\circ}$ & $+29.91^{\circ}$ \\
\hline & & $+59.49^{\circ}$ & $-60.42^{\circ}$ & $-30.29^{\circ}$ & $\pm 12.01^{\circ}$ & - \\
\hline & \multirow{2}{*}{$-10 \mathrm{~dB}$} & $\pm 39.23^{\circ}$ & $\pm 45.02^{\circ}$ & $\pm 12.77^{\circ}$ & $\pm 0.62^{\circ}$ & $+27.16^{\circ}$ \\
\hline & & $+61.29^{\circ}$ & $-58.01^{\circ}$ & $-28.25^{\circ}$ & $\pm 12.04^{\circ}$ & - \\
\hline
\end{tabular}

Table (2) shows the obtained estimated DOAs in form of eighteen roots at SNR $=0 \mathrm{~dB}$ and $\mathrm{SNR}=-10 \mathrm{~dB}$, due to that there is no need for a threshold level to limit the number of the resultant peaks in the case of using the Root MUSIC algorithms. Then, by applying the cross correlation function and using the APA algorithm for adjusting the weights of the antenna elements, the accuracy of the estimated DOA is significantly improved. 


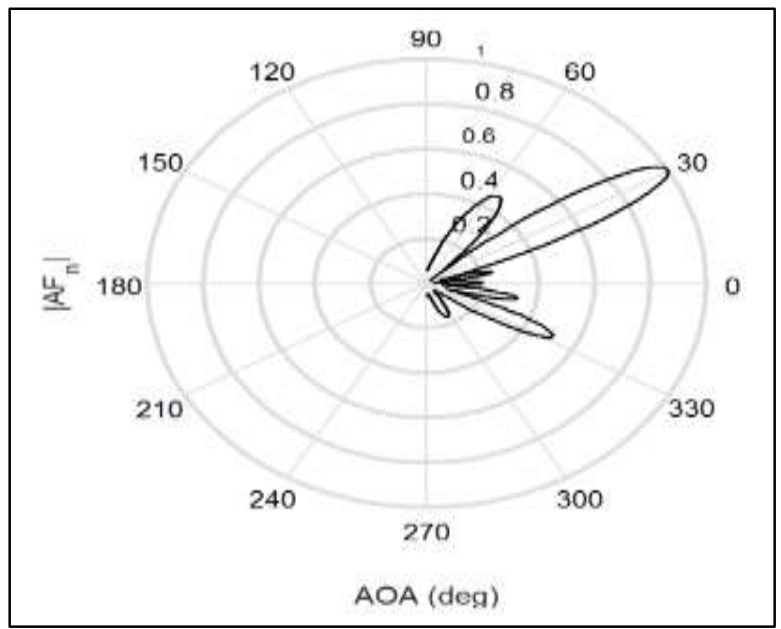

a. Polar Coordinate.

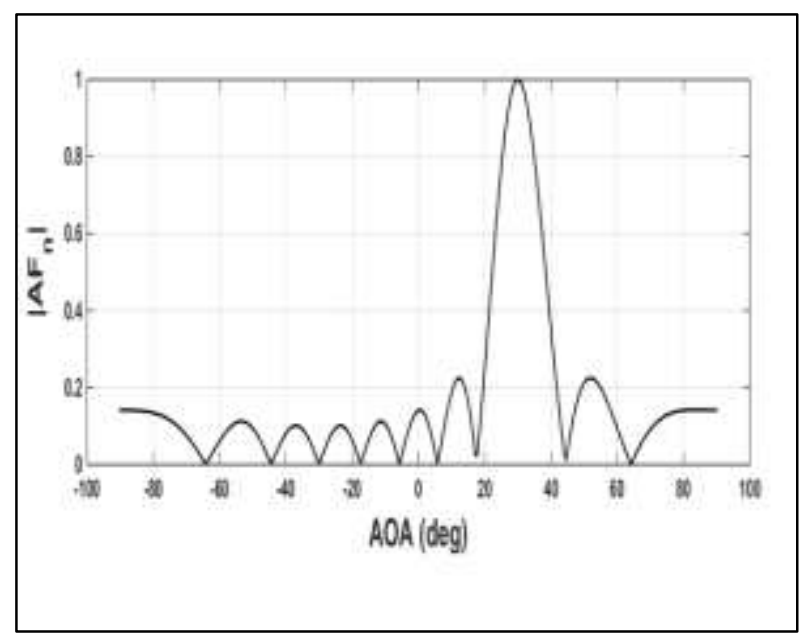

b. Cartesian Coordinate.

Figure(4): Generation of a main beam towards the desired user and nulls towards the interfering users.

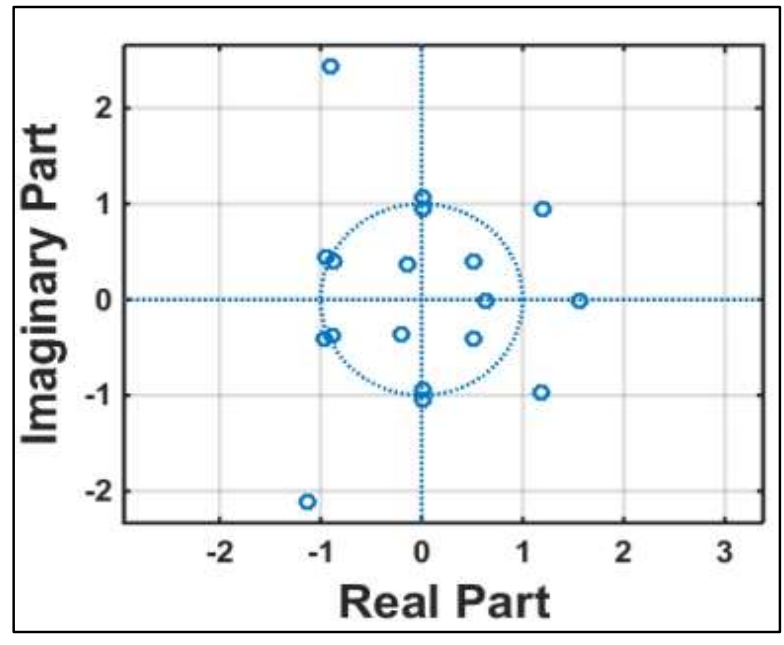

a. $S N R=O d B$.

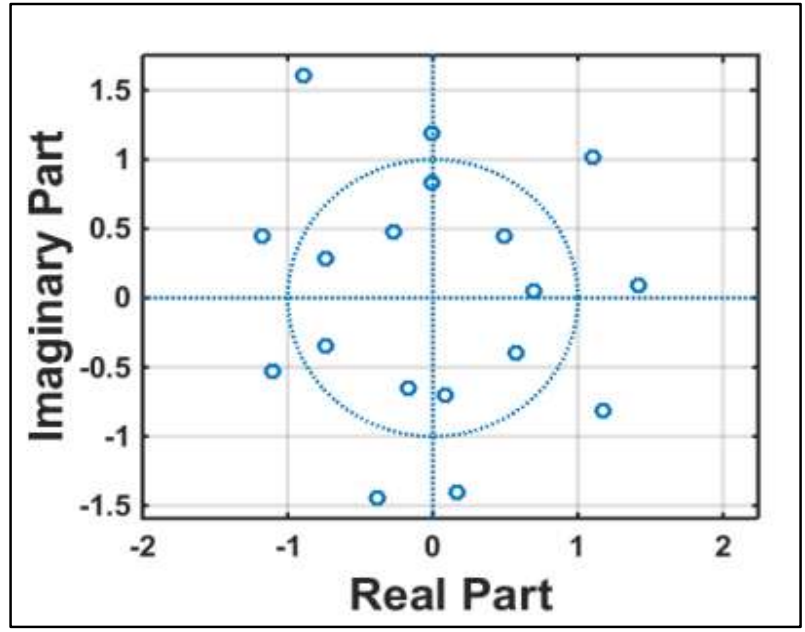

b. $S N R=-10 d B$

Figure(5): Angles associated with the Root Music Algorithm.

\section{Conclusions:}

The implementation of SDMA is the key element to meet the exponentially increasing demand on the limited resources in mobile communications. Knowing the direction of the users provides the possibility of using scheduling and frequency reuse techniques more efficiently. 
In this paper, a novel method has been introduced to identify the desired user for SIMOOFDM systems from the interfering signals. This was achieved by applying the cross correlation concept between the received signals and the reference signal which is known by the transmitter and receiver. Also, by implementing a fast adaptive processor unit such as the APA algorithm which has a fast convergence rate with minimum square error, the accuracy of the obtained DOA of the desired user was improved by determining the optimum complex weights. This in turn maximizes the signal to the interference ratio via suppressing the signal from the interference sources. The beamforming technique has been used to generate a beam towards the desired user and a null in the directions of interferes. At low SNR below $0 \mathrm{~dB}$ the accuracy of the MVDR, MUSIC, and the Root MUSIC algorithms is degraded but our simulation results show that after applying the fast adaptive APA algorithm, the performance of the DOA algorithms was improved.

\section{References:}

[1]E. M. Shaheen, "Narrowband interference suppression in DS-UWB communication systems using Digital Delay Filter," 2012 8th International Wireless Communications and Mobile Computing Conference (IWCMC), Limassol, 2012, pp. 585-590, doi: 10.1109/IWCMC.2012.6314269

[2]A. F. Molisch, Wireless communications. John Wiley \& Sons, 2011.

[3]B. Praveen and S. Das, "MIMO-OFDM Channel Estimation using Pilot Carries," International Journal of Computer Applications.

[4]L. Lingjia, et al. "Downlink MIMO in LTE-Advanced: SU-MIMO vs. MU-MIMO," IEEE Communications Magazine 50.2: pp. 140-147, 2012.

[5]F. Borko, and S. A. Ahson, eds., Long Term Evolution: 3GPP LTE radio and cellular technology, 2016.

[6]A. B. Constantine, A. B., Antenna theory analysis and design, MICROSTRIP ANTENNAS, third edition, John Wiley \& Sons, Inc., Hoboken, New Jersey, 2005.

[7] T. Tomoki, A. W. Black, and K. Tokuda. "Voice conversion based on maximumlikelihood estimation of spectral parameter trajectory." IEEE Transactions on Audio, Speech, and Language Processing 15.8, pp. 2222-2235, 2007.

[8]D. Tanee, et al., " Localization based on standard wireless LAN infra-structure using MIMO-OFDM channel state information," EURASIP Journal on Wireless Communications and Networking, pp. 1-16, 2016.

[9] S. N. Shahab, et al., "Evaluating the Impact of SNOIs on SINR and Beam pattern of MVDR Adaptive Beam-forming Algorithm," Indian Journal of Science and Technology.

[10]G. Bartoli, R. Fantacci, D. Marabissi, and M. Pucci, "LTE-a Femto-Cell Interference 
[11]Mitigation with MUSIC DOA Estimation and null Steering in an Actual Indoor Environment,", IEEE International Conference on Communications (ICC), pp. 27072711, 2013.

[12] Y. A Abohamra, A. A Amin, M. R., Solymani, and Y. R. Shayan, "Direction of Arrival algorithms for user identification in cellular networks," In Information Technology, Electronics and Mobile Communication Conference (IEMCON), 2016 IEEE 7th Annual, pp. 1-7, 2016.

[13] B. Faouzi, S. Affes, and A. Stéphenne "DOA Estimation for ULA Systems from Short Data Snapshots: an Annihilating Filter Approach," Global Telecommunications Conference, GLOBECOM 2011.

[14] S. A Napoleon, et al., "Location Estimation via the Direction of Arrival Techniques Based on the IEEE 802.11 n WLANs," International Journal of Research in Wireless Systems.

[15] M. Samir, E. M. Shaheen, and A. A. El Wahab, "Performance Analysis of DSCDMA System using Fast Adaptive Filtering under Different Jamming Techniques," in Engineering and Technology (ICET), International Conference on, pp. 1-6, IEEE, 2012.

[16] N. Sonbolestan and S. A. Hadei, "A fast affine projection algorithm based on matching pursuit in adaptive noise cancellation for speech enhancement," in 2010 International Conference on Intelligent Systems, Modelling and Simulation, pp. 193-198, January 2010. 